\title{
Elementos que influyen en el proceso de toma de decisiones en deportes individuales de alto rendimiento: un estudio cualitativo \\ Elements that influence the process of decision-making in high-performance individual sports: a qualitative study \\ *Alejandro Almonacid-Fierro, **Miguel Martinez Romero, ***Manuel Almonacid Fierro

\author{
*Universidad Católica del Maule (Chile), ** Universidad de Talca (Chile), *** Universidad Autónoma de Chile (Chile)
}

\begin{abstract}
Resumen. El estudio tiene como objetivo, identificar los elementos que influyen en el proceso de toma de decisión en deportistas de alto rendimiento, durante el momento de la competición, desde la mirada subjetiva de los propios atletas. Existirían principalmente dos teorías que explicarían la toma de decisión, por un lado, el modelo de procesamiento de la información y, por otro lado, la dimensión ecológica. No obstante, en ambas estarían involucrados una diversidad de elementos que generan como producto final una determinada decisión, generando una conducta intencional y, por ende, una consecuencia que puede ser negativa o positiva. El estudio consideró a doce deportistas de Natación, Ciclismo y Canotaje. La investigación tuvo un enfoque cualitativo utilizando una metodología de recolección de datos mediante el método inductivo realizado a través de una entrevista semi-estructurada. Los datos se analizaron mediante la codificación abierta circunscrita a la teoría fundamentada en los datos, que permite el análisis de contenido mediante fases de organización y sistematización. Como conclusión del estudio, se puede señalar que la toma de decisión en el deporte es un proceso, en donde se conjugan aspectos tales como autoconfianza, motivación, expectativas de autoeficacia, concentración y emociones, con sus respectivas subcategorías. La elección tomada por el deportista sería el punto final de todo el proceso de toma de decisión, donde debe analizar la información interna como las variables del ambiente, el momento de cada competencia, las características de cada deporte y los aspectos cognitivosemocionales que emergen en tan decisiva instancia deportiva.
\end{abstract}

Palabras claves: Toma de decisión, alto rendimiento, procesamiento de la información, dimensión ecológica.

Abstract. The main objective of the study is to identify the elements that influence the decision-making process in high-performance athletes during de time of competitions, from de subjective view of the athletes themselves. There would be mainly two theories that would explain the decision making process, on de one hand, the information processing model and the other hand the ecological dimension. However, in both of them a diversity of elements would be involved that generate as a final product a certain decision, generating an intentional behavior and, therefore, a consequence that can be negative or positive. The study considered twelve athletes from Swimming, Cycling and Canoeing. The research had a qualitative approach using a data collection methodology by means of the inductive method carried out through a semi-structured interview. The data was analyzed through open coding limited to the Theory based on the Data, which allows content analysis through organizational and systematization phases. As conclusion of the study, it can be noted that decision-making in sport is a process, where aspects such as self-confidence, motivation, expectations of self-efficacy, concentration and emotions are combined with their respective subcategories. The choice made by the athlete would be the end point of the entire decision-making process, where he must analyze the internal information such as the environmental variables, the moment of each competition, the characteristics of each sport and the cognitive-emotional aspects that emerge in such a decisive sports instance. Keywords: Decision making, high performance, information processing, ecological dimension.

\section{Introducción}

Los modelos actuales de Planificación deportiva, enfatizan la idea de ver al deportista como un individuo, respecto a sus condiciones socio-afectivas, cognitivas y condicionales, verificado eficientemente en los métodos de preparación en deportes individuales y más recientemente, adoptado por deportes colectivos (Velázquez, Reyes, \& Bolívar, 2019).

Los deportes individuales tales como natación, ciclismo y canotaje presentan una importante preparación de base en aspectos físicos, tácticos, técnicos y psicológicos. Este último elemento estaría implicado en una serie de procesos del deportista, entre ellos la toma de decisiones aspecto poco abordados en deportes individuales. Por ello es que nos resulta relevante aportar a este conocimiento, por cuanto la toma de decisiones es un proceso individual del deportista, que influye en su resultado final, afectando su desempeño y el de sus compañeros en caso de los deportes de equipo (Costa, 2018).

Fecha recepción: 23-08-19. Fecha de aceptación: 10-12-19 Aquiles Alejandro Almonacid-Fierro aalmonacid@ucm.cl
Dentro del deporte, la toma de decisiones constituye un proceso de alta complejidad, debido a que sería una variable que combinaría tanto elementos cognitivos como afectivos (Bedregal, 2010). Pero, además, hay visiones que destacan que el contexto y la interacción con el propio deportista también tendrían una incidencia importante dentro de este proceso, así como la estrecha relación entre autoeficacia, motivación y esfuerzo (Avilés, Ruiz-Pérez, Navia, Rioja, \& SanzRivas, 2014; Hernández, \& Jiménez, 2016).

Producto de que la toma de decisiones sería un proceso complejo, es importante que el deportista aprenda herramientas psicológicas que le permitan autorregularse para elegir las mejores opciones que le ayuden a tomar decisiones de manera más eficiente dentro de cada competencia que enfrenta, por este motivo, es que se consideró importante poder conocer los elementos psicológicos que influyen en éste proceso desde la perspectiva del propio deportista (González, 2017).

De acuerdo a los trabajos investigativos de Vázquez-Diz, Morillo-Baro, Reigal, Morales-Sánchez, \& HernándezMendo, (2019), durante los últimos años, el análisis de la toma de decisiones ha ganado gran interés con la publicación de diferentes investigaciones y serían varios los estu- 
dios que se han realizado en deportes colectivos como el balonmano, el voleibol y el fútbol. En algunos estudios, en particular el futbol, se ha estudiado la influencia de los factores contextuales en la toma de decisiones de jugadores altamente calificados a través de entrevistas semiestructuradas (Levi \& Jackson, 2018).

En la literatura consultada se menciona que existirían dos teorías principales que buscan explicar el proceso de toma de decisiones. Por un lado, está el modelo basado en el procesamiento de la información y, por otro lado, la dimensión ecológica (Carvallo, Araujo, Gracia \& Iglesia, 2011).

El modelo de procesamiento de la información señala que la mente humana sería un sistema que operaría a partir de símbolos, que se codifican y se almacenan para ser recuperados con posterioridad (Serrano \& Pons, 2011). Esta teoría agrega además, que existirían estrategias de análisis y procesamiento de los datos provenientes del ambiente. Una de ellas serían las estrategias de microanálisis, fundamentales en la resolución de tareas y por consiguiente, para tomar las decisiones necesarias en la solución de las problemáticas que propone una competencia deportiva. Para ello sería importante considerar también elementos como la experiencia del sujeto, el desarrollo evolutivo y cognitivo del individuo y la relación de éste con la ejecución de la tarea, pero también exige reconocer que existe una tarea que hay que resolver, para que luego el deportista pueda planificar la estrategia más adecuada. Este proceso finaliza con la evaluación a partir de las consecuencias de cada decisión para verificar si fue adecuada o no para la situación (Sáiz, Carbonero-Martín \& Flores, 2010).

Hay que mencionar, además, que éste modelo está basado en el enfoque de la psicología cognitiva, que señala que en el procesamiento de la información estarían involucradas la memoria de trabajo y estructuras de conocimiento, así como también la habilidad para anticipar las consecuencias de cada decisión o las posibles respuestas del ambiente. Todo lo anterior, depende de los órganos de los sentidos que participan en la percepción de los estímulos presentes en el entorno, para luego ser procesada e interpretada en estructuras superiores, desde donde surgirían los pensamientos e ideas acerca de las situaciones que ocurren alrededor del individuo, generando afectos y emociones. Estos elementos influirán en el acto decisional que se produciría antes de la acción, en base al procesamiento e interpretación de la información (García-González,Araujo, Carvalho \& Del Villar, 2011).

Por otro lado, la dimensión ecológica explicaría como los individuos perciben la información del entorno y cómo regulan sus movimientos, de acuerdo con la interacción que establecen con el contexto, sin necesidad de procesos internos de inferencia más allá de la percepción (Carvallo, et al., 2011). Es decir, ocurriría un proceso de percepción directa en donde el deportista es capaz de detectar la información ambiental a partir de la información útil que existe en el entorno, sin necesidad de las representaciones mentales, generándose un ajuste entre lo que es percibido y la realidad (IbáñezGijón, Travieso \& Jacobs, 2011).

Esta perspectiva se basa en la Teoría de la Percepción Directa de Gibson (1979) que postula que la información sería específica de las propiedades del entorno y la percepción es específica a la información, En este sentido, este autor plantea que existirían elementos que se mantendrían constantes frente a los cambios producidos en el ambiente o por el propio deportista, los que tienen el nombre de invariantes. Otro concepto importante planteado por Gibson, (1979), son las posibilidades de acción que se refieren a las posibles acciones que ofrecería el ambiente. En consecuencia, la toma de decisiones desde esta perspectiva sería un proceso dinámico y dependiente de ciertas variables, por lo que estará relacionada con decisiones tomadas con anterioridad (GarcíaGonzález, et al., 2011; Araujo, Teques, Hernández-Mendo, Reigal \&Anguera, 2016).

En relación al ámbito deportivo, la toma de decisiones se basaría en elegir la opción de juego más adecuada para cada momento de la competencia, lo que le añade mayor complejidad, debido a que éste proceso se caracterizaría por la necesidad del deportista de ser rápido (presión temporal) y a la vez con la necesidad de actuar de forma técnicamente competente (exigencia de precisión), agregándose además, que debe asumir la responsabilidad del resultado que implica tomar la decisión elegida, ya sea positiva o negativa (Garcia, Ruiz \& Graupera, 2011). De esta forma el deportista es capaz de responder a las variabilidades que ocurren en el ambiente (Araujo, 2011). Pero, también ésta complejidad estaría dada por los factores técnico-tácticos, los que serían afectados por multiplicidad de situaciones y elementos que impactarían en los componentes implicados en la toma de decisiones (Guillén \& Bueno, 2016; Martín, González, Calvancanti, Chirosa \& Aguilar, 2013).

Por los motivos explicitados anteriormente, es que el presente estudio busca comprender y conocer los factores que facilitan y dificultan a los deportistas de alto rendimiento tomar decisiones durante la competencia. En consecuencia, el propósito es develar cuales son las características cognitivas y del contexto, implicados en el proceso de toma de decisiones de cada deportista en situación de competencia. Cabe señalar que, a diferencia de los estudios referidos precedentemente, la presente investigación aborda la problemática de la toma de decisiones en deportes individuales, particularmente en atletas provenientes de natación, canotaje y ciclismo.

\section{Material y método}

El estudio adopta como opción metodológica la Metodología Cualitativa, toda vez que la investigación pretende acercarse a la comprensión subjetiva que tienen los sujetos en relación a un hecho, suceso, situación, temática, símbolos y objetos determinados. Vale decir, como los deportistas significan, representan y de alguna manera dan sentido a los elementos que influyen en el proceso de toma de decisión durante la competencia. El presente trabajo es un estudio fenomenológico, en el marco del paradigma interpretativo y seguidamente la metodología utilizada es la cualitativa, toda vez que pretende comprender, describir y analizar el proceso de toma de decisiones en deportistas de alta competencia (Gibs, 2012; Flores, 2009).

\section{Participantes}

En la tabla ${ }^{\circ} 1$ (ver anexos), se caracteriza la muestra que participó del estudio, el que consideró a 12 deportistas, siete 
hombres y cuatro mujeres cuyas edades oscilaban entre 17 a 28 años de edad del Centro de Entrenamiento Regional (CER) de la Región del Maule- Chile, en los deportes de Natación clásica, Ciclismo de pista y Canotaje de velocidad, la cual tuvo como criterio de exclusión que hayan obtenido logros importantes en las últimas competencias, a saber, en torneos sudamericanos o superior con resultados de medalla, a esto lo llamamos nivel competitivo internacional.

\begin{tabular}{|c|c|c|c|c|}
\hline \multicolumn{5}{|c|}{ ización de la mue } \\
\hline 1 & Canotaje de velocidad & 21 & Masculino & Internacional 6 años \\
\hline 2 & Canotaje de velocidad & 28 & Masculino & Internacional 13 años \\
\hline 3 & Canotaje de velocidad & 21 & Masculino & Internacional 6 años \\
\hline 4 & Canotaje de velocidad & 28 & Femenino & Internacional 13 años \\
\hline 5 & Canotaje de velocidad & 22 & Masculino & Internacional 7 años \\
\hline 6 & Canotaje de velocidad & 22 & Masculino & Internacional 7 años \\
\hline 7 & Canotaje de velocidad & 21 & Masculino & Internacional 6 años \\
\hline 8 & Natación clásica & 19 & Masculino & Internacional 4 años \\
\hline 9 & Natación clásica & 23 & Masculino & Internacional 8 años \\
\hline 10 & Natación clásica & 17 & Femenino & Internacional 2 años \\
\hline 11 & Ciclismo de pista & 21 & Femenino & Internacional 6 años \\
\hline 12 & Ciclismo de pista & 21 & Masculino & Internacional 6 años \\
\hline
\end{tabular}

\section{Instrumento}

En esta fase de la investigación, se optó por la entrevista semiestructurada como recurso metodológico por sus características flexibles, en el bien entendido que la técnica se comprende como un proceso interactivo entre el investigador y el entrevistado, en consecuencia, la entrevista semiestructurada favorece el habla de los participantes, dándoles la posibilidad de reflexionar y conversar libremente acerca de sus experiencias de vida (Kvale, 2011; Bohórquez \& Checa, 2017).

\section{Procedimiento}

Lo anterior, se realizó a través de los relatos de las experiencias de los propios deportistas, en específico, se realizó un análisis de contenido a través de la comparación constante, circunscrito a la Teoría Fundamentada (Glaser \& Strauss, 1967). De esta forma, fue posible acceder a los procesos subjetivos que se dan en la toma de decisiones, expresados a través de los diálogos de cada deportista, posibilitando realizar una comparación entre cada uno de ellos, analizando cuales son los factores implicados en el proceso de toma de decisiones durante una competencia, a través de la verbalización por parte del mismo deportista. Los datos fueron trabajados a través del software Nvivo 10.

\section{Tipo de estudio y diseño}

Considerando que los diseños de investigación deben transitar en ámbitos flexibles, en términos de enfrentar campos de acción impredecibles, la investigación se estructuró en las siguientes fases

Fase 1: Definición de la muestra: se realizó a través del muestreo teórico.

Fase 2: Validación del guion de entrevista: el guion de entrevista fue elaborado por el equipo de investigadores y validado por un panel de tres académicos universitarios.

Fase 3: Aplicación de las entrevistas: esta fase se realizó durante los meses de octubre y noviembre del año 2017, tuvieron una duración de 60 minutos y fueron aplicadas por los investigadores responsables.

Fase 4: Transcripción y análisis: una vez transcritas las entrevistas se sometieron a análisis de contenido

Fase 5: Fase de codificación y categorización: se reali- zó una codificación abierta, cuestión que hizo emerger de los datos un gran abanico de códigos, y seguidamente posibilitó ir integrando los códigos en unidades mayores denominadas categorías secundarias, para luego agruparlas en categorías primarias, estableciendo las correspondientes relaciones con la pregunta de investigación, cuidando la exclusividad de las categorías, de manera que no haya habido solapamiento de ellas (Almonacid-Fierro, Merellano-Navarro, Feu, Vizuete, \& Orellana, 2019).

Fase 6: Interpretación: una vez que se sistematizaron los datos en una matriz, se procedió al proceso de interpretación en base a la teoría fundamentada (Glaser \& Straus, 1967).

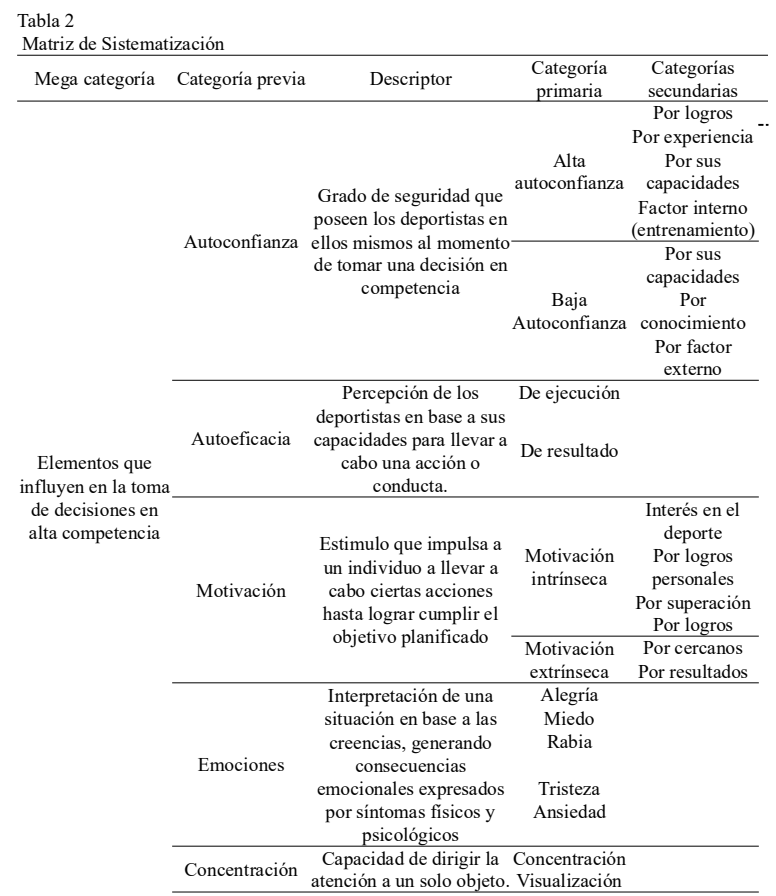

\section{Técnica de Recolección de datos}

La técnica utilizada en el presente estudio fue la entrevista semiestructura, toda vez que representa una metodología ampliamente utilizada en aquellas investigaciones que pretenden acercarse a la comprensión del sentido y significado de realidades humanas diversas. En consecuencia, la entrevista semiestructurada favorece el habla de los participantes, dándoles la posibilidad de reflexionar y conversar libremente acerca de sus experiencias de vida (Kvale, 2011; Taylor y Bogdan, 1984). El procedimiento para determinar la validez del instrumento se realizó mediante el juicio o evaluación de experto. La entrevista fue validada por tres académicos universitarios.

\section{Criterios de Rigor o de Calidad}

En la investigación cualitativa se utilizan criterios de fiabilidad y validez a objeto de asegurar la calidad del estudio y consecuentemente que los datos recogidos, primeramente, y luego analizados e interpretados, cuentan con criterios de calidad, vale decir que hayan sido sistematizados y sometidos a rigor metodológico. En este contexto el presente estudio recurrió a los siguientes criterios de calidad, expuestos por (Flick, 2012; Flores, 2009): credibilidad; obtención de datos ricos; compromiso con el trabajo de campo; confirmabilidad. 


\section{Resultados y análisis de los datos}

Se aplicaron 12 entrevistas siguiendo las recomendaciones de (Flores, 2009; Kvale, 2011; Taylor \& Bogdan, 1984). Las entrevistas se trabajaron bajo la perspectiva teórica de la teoría fundamentada Coffey \& Atkinson, (2003); Strauss \& Corbin, (2002) y Gibbs, (2012). En este sentido se levantó en un primer momento una gran categoría que permitiera agrupar a las demás, y seguidamente tres categorías previas que emergen del marco teórico de la investigación y que orientan el trabajo de análisis y reducción de datos posterior, de tal manera que la primera parte de la matriz se conformó con los siguientes elementos teóricos:

Megacategoría: Toma de decisiones en el alto rendimiento: esta categoría da cuenta del problema de investigación, y es el constructo teórico que permite ir tejiendo el discurso de los deportistas.

Categorías previas: emergen desde el marco teórico de la investigación y son las siguientes: autoconfianza, autoeficacia, motivación, emociones y concentración.

Categorías primarias: surgen desde el proceso de reducción de datos de las categorías secundarias

Categorías secundarias: emergen a partir del análisis del proceso de codificación.

En este apartado se presentan los resultados obtenidos a partir del análisis cualitativo descriptivo, realizado a partir de la revisión y categorización de las respuestas entregadas por los deportistas que fueron entrevistados, dando origen a la codificación abierta (Coffey \& Atkinson, 2003).

Las categorías utilizadas se definieron a partir de la revisión de material bibliográfico sobre los elementos que influyen en la toma de decisiones en deportistas de alto rendimiento, ajustando a cada concepto los diálogos recogidos en cada una de las entrevistas, para luego elaborar conclusiones y determinar cuáles elementos son los que afectan tanto positiva como negativamente, la toma de decisiones durante una competencia en deportistas de alto rendimiento y luego realizar el análisis cualitativo respectivo.

Los resultados que a continuación se presentan muestran los factores que influyen en un deportista de alto rendimiento a la hora de tomar decisiones durante una competencia. En este sentido se identificaron los siguientes elementos: autoconfianza, autoeficacia, motivación, emociones, y concentración. Para mostrar el impacto de estos factores se citan comentarios textuales que fueron clasificados en las categorías respectivas más representativas, en base a las respuestas dadas por los deportistas.

La figura $\mathrm{N}^{\circ} 1$, muestra los elementos que favorecen o facilitan la toma de decisiones. Se puede observar que la autoconfianza influiría de forma positiva en el proceso de toma de decisiones recordando logros anteriores, debido a que éstos entregarían un antecedente al deportista de que es posible alcanzar los resultados esperados, así como también la sensación de experiencia, lo que le permite confiar en sus cualidades, esto impactaría en las expectativas de autoeficacia, relacionándose de esta manera con emociones de alegría, que podría generar motivación intrínseca.

En la figura $\mathrm{N}^{\circ} 2$ se muestran los elementos que influirían negativamente en el proceso de toma de decisiones, aquí estarían la baja autoconfianza, la que podría ser resultado de

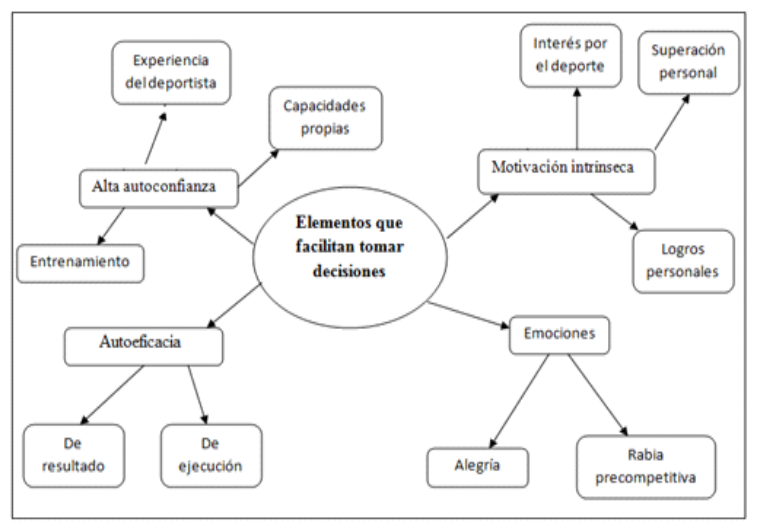

Figura $n^{\circ} 1$ elementos que favorecen o facilitan la toma de decisiones. Fuente: elaboración propia falta de experiencia del deportista a la hora de enfrentar una situación determinada dentro de la competencia, así como también las condiciones ambientales, tales como temperatura, viento, presión del ambiente, por nombrar algunas, pueden incidir en la creencia del atleta en las herramientas personales que posee, lo cual podría influir en el aumento de la ansiedad producto que su atención y concentración se centrarían en elementos externos que no son controlables por el propio deportista, influyendo en lo descrito anteriormente, lo cual lo llevaría a una motivación principalmente extrínseca tal como, conseguir de forma rápida el resultado deseado.

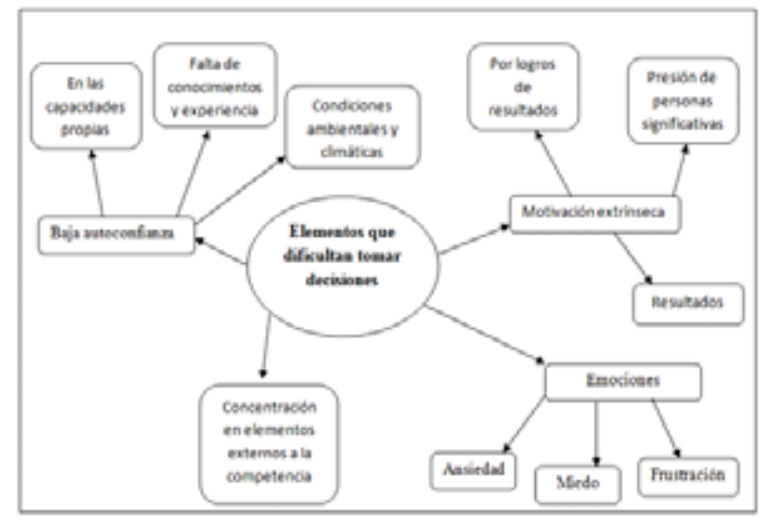

Figura $n^{\circ} 2$ elementos que dificultan la toma de decisiones. Fuente: elaboración propia

A continuación se explicitan y se detallan, los elementos que intervienen en la toma de decisiones generados a partir de la categorización realizada mediante la entrevista semiestructurada a los deportistas de alto rendimiento respaldado por la revisión bibliográfica para definir éstos elementos (autoconfianza, autoeficacia, motivación, emociones, y concentración), a continuación se ejemplifican con diálogos obtenidos de las entrevistas realizadas a cada deportista, las que se citan textualmente.

\section{Autoconfianza}

La autoconfianza se comprende como el grado de seguridad que poseen los deportistas en ellos mismos al momento de tomar una decisión en competencia. El nivel de autoconfianza puede ser alto o bajo Es así como, la experiencia del deportista [ «Influye harto, de manera que me siento más decidido, siento que puedo dar todo lo de mí, me siento preparado psicológicamente, mayor madurez deportiva que me ayuda en la competencia»] como también de la percep- 
ción positiva de sus capacidades [«Normalmente no, como que mi autoconfianza me ayuda a competir porque eh me siento capaz de las condiciones que puedo tener y no tengo duda de lo que puedo ser»] y la calidad del entrenamiento realizado para enfrentar cada competencia [«Eso según yo influye mucho, porque según lo que entreno y como lo hago entrenando me baso en como lo voy a hacer compitiendo, porque si me preocupo aquí de hacer bien las vueltas para el momento de la competencia hacerlas bien, la salida y todo eso»], éstos factores estarían relacionados con un alto nivel de autoconfianza.

Sin embargo, un menor nivel de experiencia del deportista [«Casi siempre con las pruebas que no he nadado, que como lo voy a hacer porque si no tengo experiencia en eso me complica con lo que voy a hacer»], el conocimiento que tenga sobre su deporte o los rivales a enfrentar [«No es por mis capacidades sino es como no sé si el resto va a funcionar por las cosas de afuera, como no sé si los otros corredores van a estar mejor que yo, pero yo confió en mis capacidades porque también he entrenado para algo «] y factores externos como el clima o la influencia de personas significativas [«lo mismo el viento que haya, los tipos que compitan los veo como llegan, si son grandes o cosas así, si están remando bien, como me sienta en el agua, como sea la sensación en el bote»] estarían relacionados con un nivel bajo de autoconfianza. Por lo tanto, el nivel de autoconfianza tendría influencia en el proceso de toma de decisiones.

\section{Autoeficacia}

Por su parte, la autoeficacia sería otra variable que intervendría en la toma de decisión, pues se define como la percepción que poseen los deportistas en base a sus capacidades para llevar a cabo una acción o conducta necesaria para lograr un resultado deseado. Dentro de esta variable existe la autoeficacia de ejecución y autoeficacia de resultad, teniendo en cuenta las posibilidades y probabilidades que la decisión tomada genere un resultado deseado estaría relacionado expectativas de autoeficacia de ejecución [«Eh al momento que decido actuó nomas, decido y actuó»], sin embargo, también podría haber una baja percepción por parte del deportista de lograr ejecutar adecuadamente los movimientos y pasos necesarios para lograr tomar una decisión de manera adecuada para enfrentar las situaciones que se enfrenta en cada competencia [«En las pruebas grupales las subidas porque yo no me siento muy segura, no me destaco mucho en subidas eso como que si es una carrera con subidas me va a poner presión»] pero también existen expectativas de autoeficacia asociadas al resultado, factor que también incluye una graduación, así tenemos que puede haber una alta expectativa de autoeficacia de logro [«es más difícil con competidores conocidos que desconocidos porque sé que pueden estar mejor que yo, en cambio en las internacionales especialmente que no los conozco, voy a luchar con lo que tengo solamente»] y una baja expectativa de autoeficacia asociada al resultado[«En la salida porque sé lo que tengo que hacer, en realidad ya llegando a la meta eh ahí se me hace más complicado porque siento esa incertidumbre de que va a pasar»] que de todas formas influirá en el proceso de toma de decisiones del deportista durante la competencia.

\section{Motivación}

Otro factor importante que influye en la toma de decisiones es la motivación, estimulo que impulsa a un individuo a llevar a cabo ciertas acciones manteniendo firme su conducta, hasta lograr cumplir el objetivo planteado. La fuente de motivación, basado en la Teoría de la Autodeterminación (Stover, Bruno, Uriel, \& Liporace, 2017), puede ser extrínseca o intrínseca. En este sentido la motivación intrínseca se encontró que está relacionada con el interés por el deporte que practica ocuparla con más ganas y ocuparla en el deporte, igual en el tema del deporte hay que ser perseverante entrenar todo los días»], buscar logros personales y por superación personal [«Pensaba dar lo mejor de mí, de hacer algo nuevo que nadie había hecho, de superarme los logros anteriores más que nada superarme a mí mismo. Y durante de la competencia solo pienso en luchar y dar lo mejor de mí»»].

Mientras que la motivación extrínseca se centra en elementos que no dependen del deportista mismo tales como principalmente la obtención de logros de resultados [« más que nada los resultados casi siempre eso es lo que más me preocupa], por satisfacer necesidades o no defraudar a terceros [«La decisión la tomé yo, pero las motivaciones son externas, siempre ser el mejor, estar en este caso, darle como el ejemplo a mi hermano, darle el orgullo a mi mama a mi papa etc.., siempre han estado como presentes en todo el deporte, en lo que ha sido mi logro deportivo»].

\section{Concentración}

La concentración se refiere a la capacidad de dirigir la atención a un solo objeto, es el acto de abstraerse de todo estimulo que altere la atención del deportista. Entre los elementos que ayudan a mantener una concentración en el foco de atención correcto es la visualización [«antes de la competencia es como me mentalizo que voy a hacer en la competencia, como lo voy a hacer como no lo voy a hacer etc.»]. Sin embargo, existen otros deportistas que se centran en las metas que desean conseguir [ He me enfoco en lo que he entrenado, en lo que quiero conseguir y en lo que voy a hacer») . Mientras que hay factores que hacen que el foco de atención esté puesto en otros elementos que provocan desconcentración, como son los factores externos [«He si a veces, me desconcentro mucho en el rival por parte táctica cuando voy tanteando al rival si va más adelante o más atrás, eso es igual un tema de concentración, me despreocupo de mí, del punto donde tengo que llegar y de lo que tengo que hacer»].

\section{Emociones}

Las emociones se refieren a la interpretación que una persona (deportista en este caso) le asigna a una situación determinada en base a las creencias que podrían generar consecuencias afectivas, físicas y psicológicas. Pero como se señaló el proceso de toma de decisiones es un fenómeno cognitivo y afectivo, por ende, todos los factores hasta aquí estudiados son afectados por el estado emocional del deportista, apareciendo estados de ánimos positivos y/o negativos [«Eh si es correcta la decisión que tomé, felicidad porque tengo un buen logro pero si es mala la decisión que tomé, me da igual, un poco de enojo, pero después me resigno a no cometer de nuevo el mismo error»]. Las emociones 
que se expresan comúnmente en el deporte son Alegría [porque lo hice bien frente al éxito y frente al fracaso, un poco de frustración a veces pero hasta cierto punto, pero como para ver como lo puedo mejorar para la otra no quedarme con eso»], Frustración [Dependiendo si es un error frustración conmigo mismo...»], Miedo [ «Si porque tengo como miedo después a equivocarme en todo el resto, pero ese temor así como que lo calmo, de alguna manera así viendo otros videos de competencias mías porque ya veo como lo hago y de que no me sale tan mal y todo eso»], pena [ $\ll \mathrm{Si}$, supongo por ejemplo cuando yo no entreno eso ya afecta en una competencia porque igual ando bajoneado como por ejemplo me va a ir mal en la competencia igual eso me afecta»], Rabia [«ahí como que me enojo porque si tome esa decisión es para que me salga bien pero algunas veces fracaso y no, ya uno entra a enojarse en sí mismo pero son cosas que pasan»)].

Relacionado con lo anterior, el deportista antes de una competencia o cuando debe tomar decisiones dentro de ésta suele haber cierto nivel de miedo. Respuesta normal que se produce cuando la persona percibe una posible amenaza donde la intención es protegerse de dicha amenaza haciendo que evitemos la situación. [ «La ansiedad, el miedo, no estar bien preparado a veces, tenerle miedo al rival, eso»]

\section{Discusión}

Luego de revisar la información actualizada sobre el tema de toma de decisiones se pudo constatar que existen dos visiones principales, la teoría de procesamiento de la información y la teoría ecológica, las que buscan explicar los factores que intervienen en la toma de decisión, desde la perspectiva del propio deportista. Por un lado, la perspectiva cognitiva aporta la visión desde el modelo de procesamiento de la información, que propone que en la toma de decisión estarían involucradas la memoria de trabajo y estructuras de conocimiento, que permitirían al deportista anticipar las consecuencias de cada decisión o las posibles respuestas del ambiente (García-González, et al., 2011), Por lo tanto, sería necesario que luego el deportista pueda planificar la estrategia más adecuada, en cada momento o situación que enfrenta en la competencia (Saiz, Carbonero-Martín \& Flores, 2010).

Por otro lado, la perspectiva ecológica le asignaría un papel importante al contexto y a la interacción que se genera entre éste y el deportista, donde intervendrían variables asociadas principalmente a la percepción, en que el deportista es capaz de detectar la información ambiental a partir de la información útil que existe en el entorno, sin necesidad de las representaciones mentales (Carvallo, et al.,2011), de esta forma el deportista podría regular sus movimientos considerando la información del ambiente, pudiendo responder de manera rápida e inmediata, con gran precisión y eficacia, sin tener necesariamente que procesar la información (Avilés, et al., 2014).

En el presente estudio, se han encontrado elementos que facilitan y/o dificultan la toma de decisiones por parte de los deportistas autoconfianza, autoeficacia, motivación, emociones, y concentración. Lo anterior, tendría relación con que habría deportistas con una mayor facilidad para decidir en situaciones donde el componente racional es mayor, pero tendrían dificultades cuando el tiempo disponible para decidir es muy reducido. Otros muestran déficits perceptivos, normalmente asociados a déficits atencionales, como consecuencia del desconocimiento de cuáles son en cada momento de la competencia los índices relevantes de información de los cuales puede depender la toma de decisión correcta (Cárdenas, Perales \& Alarcón, 2015).

En este sentido, el nivel de autoconfianza no sería un elemento estable en el deportista, ya que se ve influida por las características del contexto y por las situaciones que enfrenta, lo que apoya lo planteado por la perspectiva ecológica de la toma de decisión. En consecuencia, se puede señalar que éste elemento diferenciaría a los deportistas novatos con los expertos, de hecho, la percepción de competencia decisional aumentaría a medida que el atleta incrementa su nivel competitivo (León, Boix, Serrano \& Paredes, 2017). Lo anterior, concuerda con lo encontrado en el presente estudio donde se pudo observar que el grado de autoconfianza está influido por el grado de experiencia del deportista al enfrentar situaciones, por la valoración de las capacidades propias como también de factores internos como el entrenamiento y factores externos como las condiciones ambientales, por ejemplo, que debe enfrentar en cada competencia o entrenamiento.

Otro de los elementos relevantes del estudio, que emerge a partir del relato de los sujetos investigados, dice relación con los aspectos motivacionales del deportista durante la competición, puesto que la motivación sería un elemento central en impulsar al deportista a llevar a cabo ciertas acciones, manteniéndose firme hasta lograr el objetivo planteado, aun cuando los obstáculos sean difíciles de afrontar, cuestión que se ve supeditada en ocasiones por lograr un resultado, tal como lo expresan los informantes claves en su relato. Esto concuerda con lo reportado por la literatura, que señala que, si bien tradicionalmente la toma de decisión habría sido considerada como una habilidad cognitiva, también se ha considerado que intervendrían los aspectos emocionales y que la motivación jugaría un aspecto importante dentro del proceso de toma de decisiones, tanto en entrenamientos como en la competencia (León, et al., 2017; Kumar, Singh, Sandhu, Gupta, \& Pandey, 2017; Morgan, 2017).

Por su parte, las emociones negativas como el miedo o la pena, pueden debilitar el rendimiento del deportista aumentando la ansiedad, dificultando la concentración y provocando dificultades en el proceso de la toma de decisión. Es así como, por ejemplo, el miedo podría ser una emoción que en general bloquea la toma de decisión, ya que produciría dudas y desconcentración en el deportista, lo que llevaría al aumento de la probabilidad de errores. Esto se puede respaldar con lo señalado por Maldonado, Martín-Tamayo \& Chirosa (2015), en su estudio, quién encontró que los deportistas que experimentan dificultades emocionales, tendrían menor compromiso de aprendizaje decisional, mientras que la rabia y la tristeza estarían vinculadas al resultado, por ende, puede estar asociado a la motivación por el logro de los resultados y no decepcionar a los cercanos. No obstante, el deseo de alcanzar una determinada recompensa también podría favorecer la toma de decisiones en los distintos contextos, escenarios y situaciones que enfrenta el deportista en la competencia, por ende, esto sugiere que los aspectos 
motivacionales son fundamentales para lograr el equilibrio y que en consecuencia la toma de decisión sea eficaz. Esto concuerda con que el sentirse competente y lograr resultados deseados es fundamental dentro del contexto deportivo (Melgarejo, 2015).

\section{Conclusiones}

Durante el proceso de toma de decisiones, siempre debemos identificar el problema central que se va a discutir, los hechos y circunstancias involucrados, las alternativas y sus respectivas consecuencias. En el deporte de alto rendimiento el proceso de toma de decisiones se torna complejo puesto que se deben tener en cuenta varios factores como la especialidad deportiva, el sistema de competencias, el nivel de entrenamiento, los rivales, el recinto deportivo, las condiciones climáticas, etc. En este sentido, podemos comprender el proceso de toma de decisiones como una cuestión compleja e interdependiente de varias variables que interfieren en la acción final (Araujo, 2011).

Detrás de la capacidad de un atleta para tomar decisiones hay factores involucrados que pueden interferir positiva y negativamente. Estos factores pueden ser cognitivos como la capacidad de anticipar y percibir que actuará especialmente en el tiempo que le tomará al atleta tomar la decisión. Cómo también la memoria que servirá como contribución para que los deportistas rescaten las decisiones tomadas en situaciones previamente experimentadas y almacenar las nuevas situaciones que van encontrando en el momento de competencia (Maldonado, Martin, Tamayo \& Chirosa, 2015).

En este contexto y apoyándonos en los datos analizados, se puede inferir que ambas teorías abordadas en el estudio, y que intentan explicar el proceso de la toma de decisión, tienen consistencia en sus postulados, ya que existen momentos en la competencia donde el deportista necesita procesar la información para llegar a tomar una decisión. Así como también hay momentos en que tendrá elementos y situaciones en donde debe actuar sobre el ambiente. Por ende, el contexto, también juega un rol importante en la toma de decisión como lo señala la teoría ecológica (GarcíaGonzález, et al., 2011).

A pesar que los dos modelos planteados dependerían de las variables ya mencionadas, se puede inferir a partir de los datos, que el nivel de experiencia y conocimiento de la actividad deportiva, la preparación (entrenamiento físico y mental) así como de los distintos contextos, hará que el deportista pase de procesar la información, que tendería a ser un camino más largo en la toma de decisión, hacia el proceso ecológico, que plantea este modelo en el cual disminuirán los pensamientos, lo que permitirá que el deportista actúe de forma más fluida y la toma de decisión tendrá una mayor velocidad para ser ejecutada. Esto se respalda en lo señalado por Mann, Williams, Ward \& Janelle (2007), que plantean que los deportistas expertos serían mejores en la captación de índices perceptuales, ya que tendrían períodos más prolongados de tranquilidad visual. Esto se podría explicar porque los deportistas más experimentados responderían a estrategias perceptivas deliberadas como lo señalan Aguilar, Tamayo \& Chirosa (2014), mientras que los más novatos realizarían una búsqueda visual de acuerdo al orden en que ocurrirían los eventos. Lo que haría que los deportistas con mayor experiencia sean más eficientes para interpretar la información disponible, en el ambiente, por lo tanto, la reacción y eficacia de sus decisiones sería también mayor, cuestión que concuerda con los resultados encontrados en el presente estudio.

\section{Referencias}

Abraldes, J. Granero-Gallegos, A. Baena-Extremera, A. GómezLópez, M. \& Rodríguez-Suárez, N. (2016). Orientaciones de meta, satisfacción, creencias de éxito y clima motivacional en nadadores. Revista Internacional de Medicina y Ciencias de la Actividad Física y el Deporte 16(62) 583-599.

Aguilar, J. Tamayo, I. \& Chirosa, L. (2014). Estudio de la dimensión subjetiva de la toma de decisiones en estudiantes de bachillerato mediante el Cuestionario de Estilo de Decisión en el Deporte (CETD). Revista Iberoamericana de Psicología del Ejercicio y el Deporte, 9 (1), 209-220.

Almonacid-Fierro, A. Merellano-Navarro, E., Feu, S. Vizuete, M. \& Fernández, R. (2019). Perspectiva cualitativa en la construcción del conocimiento didáctico del contenido del profesorado de Educación Física. Retos: Nuevas tendencias en educación física, deporte y recreación, 36(36), 459-468.

Araújo, D. (2011). Monográfico de innovaciones en el estudio de la toma de decisiones en el deporte. Revista de Psicología del Deporte, 20(1), 639-643.

Araújo, D. Teques, P. Hernández-Mendo, A. Reigal, R. \& Anguera, M. (2016). La toma de decisión, ¿es una conducta observable?: Discusión sobre diferentes perspectivas teóricas utilizadas en el estudio del rendimiento deportivo. Cuadernos de Psicología del Deporte, 16(1), 183-196.

Avilés, C. Ruiz-Pérez, L. Navia, J. Rioja, N. \& Sanz-Rivas, D. (2014). La pericia perceptivo-motriz y la cognición en el deporte: del enfoque ecológico y dinámico a la enacción. Anales de Psicología, 30(2), 725-737. https://dx.doi.org/ 10.6018/analesps.30.2.158611

Bedregal, P. (2010). Reflexiones en torno a las emociones en la toma de decisiones éticas en medicina. Revista Chilena de Neuropsicología, 5(1)21-30.

Bohórquez, M. \& Checa, I. (2017). Diseño y validez de contenido de una entrevista para la evaluación psicológica de porteros de fútbol. Revista de Psicología Aplicada al Deporte y al Ejercicio Físico, 2 (1), 1-12. https://doi.org/ 10.5093/rpadef2017a3

Cárdenas, D. Perales, J. \& Alarcón, F. (2015). La planificación del entrenamiento para la toma de decisiones en los deportes de equipo. El entrenamiento táctico y decisional en el deporte. Madrid: Síntesis, 264-89.

Carvallo, J. Araujo, D. Gracía, L. \& Iglesia, D. (2011). El entrenamiento de la toma de decisiones en el tenis: ¿Qué fundamentos científicos se pueden aplicar en los programas de entrenamiento? Revista Psicología del Deporte, 20 (2), 767-783.

Coffey A. \& Atkinson P. (2003). Encontrar el sentido a los datos cualitativos. Estrategias complementarias de in- 
vestigación. Medellín: Universidad de Antioquia.

Costa, D. (2018). Processo de tomada decisão e esporte: aspectos bioéticos no futebol. Universidade Federal do Rio Grande do Sul. Porto Alegre.

Flick, U. (2012). Introducción a la investigación cualitativa. Madrid: Ediciones Morata.

Flores R. (2009). Observando observadores: una introducción a las técnicas cualitativas de investigación social: Santiago: Ediciones UC.

García, V. Ruiz, L. \& Graupera J. (2011). Perfiles decisionales de jugadores y jugadoras de voleibol de diferente nivel de pericia. RICYDE. Revista Internacional De Ciencias Del Deporte. 5(14), 123-137. Doi:10.5232/Ricyde,

García-González, L. Araujo, D. Carvalho, J \& Del Villar, F. (2011). Panorámica de las teorías y métodos de investigación en torno a la toma de decisiones en el tenis. Revista Psicología del Deporte, 20(2), 645-666.

Gibson, J., (1979). The Ecological Approach to Visual Perception, New York. Taylor \& Francis Group.

Gibbs, G. (2012). El análisis de datos en investigación cualitativa. Madrid. Ediciones Morata.

Glaser, B., \& Strauss, A. (1967). The Discovery of Grounded Theory: Strategies for Qualitative Research. Chicago: Aldine Publishing Company.

González, J. (2017). Diseño del entrenamiento mental del tenista. De lo científico a la aplicado. Revista de Psicología aplicada al deporte y al ejercicio físico, 2(1), 1-14.

Guillén, L. \& Bueno, E. (2016). Incidencia de la contextualización en la adquisición de los elementos técnicos - tácticos del Karate Do durante la iniciación deportiva. Retos: Nuevas Tendencias en Educación Física, Deporte y Recreación, (30), 59-63.

Hernández, J. \& Jiménez, A. (2016). Personalidad y respuesta psicológica en deportistas. Representación temporal y adaptativa del proceso persona-deporte. Retos: Nuevas tendencias en educación física, deporte y recreación, (30), 211-215.

Ibáñez-Gijón, J. Travieso, D. \& Jacobs, D. (2011). El enfoque neogibsoniano como marco conceptual y metodológico para el diseño de programas de entrenamiento deportivo. Revista de Psicología del Deporte, 20 (2), 667-688.

Kvale S. (2011). Las entrevistas en investigación cualitativa. Madrid: Ediciones Morata.

Kumar, V. Singh, A. Sandhu, J. Gupta, N. \& Pandey, R. (2017). Comparative study of sports motivation among contact and non-contact sports persons. International Journal of Physical Education, Sports and Health, 4(4), 128-131.

Levi, H. \& Jackson, R. (2018). Contextual factors inûuencing decision making: perceptions of professional soccer players. Psychol. Sport. Exerc. 37, 19-25. doi:10.1016/ j.psychsport.2018.04.001

León, E. Boix, S. Serrano, M. \& Paredes, N. (2017). Motivación y toma de decisiones en voleibol: influencia de los años de experiencia. Revista de Psicología aplicada al deporte y al ejercicio físico. 1(4) 1-10. DOI: https:// doi.org/10.5093/rpadef2017a4

Mann, D. Williams, A. Ward, P. \& Janelle, C. (2007). Perceptualcognitive expertise in sport: A meta-analysis. Journal of Sport and Exercise Psychology, 29(4), 457-478.

Maldonado, L. Martín-Tamayo, I. \& Chirosa, L. (2015). Aná- lisis de las características psicológicas y la toma de decisiones en un grupo de nadadores. Revista Iberoamericana de Psicología del Ejercicio y el Deporte, 10 (1), 4955 .

Martín, I. González, A. Cavalcanti, L. Chirosa, 1. \& Aguilar, J. (2013). Fiabilidad y optimización del programa PROTODEBA v 1.0 para la observación de la Toma de Decisiones en Balonmano. Cuadernos de Psicología del Deporte, 13(1) 63-70. doi.org/10.4321/S157884232013000100007

Melgarejo, A. (2015). Relación entre inteligencia emocional y motivación de logro en deportistas de alto rendimiento. Tesis Doctoral. Universidad Argentina de la Empresa.

Morgan, K. (2017). Reconceptualizing motivational climate in physical education and sport coaching: An interdisciplinary perspective. Quest, 69(1), 95-112.

Sáiz, M. Carbonero-Martín, M. \& Flores, L. (2010). Análisis del procesamiento en tareas tradicionalmente cognitivas y de teoría de la mente en niños de 4 y 5 años. Psicothema, $22(4), 772-777$.

Stover, J. Bruno, F. Uriel, F. \& Liporace, M. (2017). Teoría de la Autodeterminación: Una revisión teórica. Perspectivas en Psicología, 14(2), 105-115.

Serrano, J. \& Pons, R. (2011). El Constructivismo hoy: enfoques constructivistas en educación. Revista electrónica de investigación educativa, 13(1), 1-27.

Strauss, A. \& Corbin, J. (2002). Bases de la investigación cualitativa. Técnicas y procedimientos para desarrollar la teoría fundamentada. Medellín: Editorial Universidad de Antioquia.

Taylor, S. \& Bogdan R. (1984). Introducción a los métodos cualitativos de investigación. Barcelona: Editorial Paidós.

Vázquez Diz J. Morillo Baro, J. Reigal, R. Morales-Sánchez, V. \& Hernández-Mendo, A. (2019). Contextual factors and decision-making in the be-haviour of finalization in the positional attack in beach handball: diferences by gender through polar coordinates analysis. Front. Psychol. 10 1386 | doi: https://doi.org/10.3389/fpsyg.2019.01386

Velázquez, J. Reyes, N. \& Bolívar, N. (2019). Revisión teórica de la planificación tradicional y contemporánea en el entrenamiento deportivo. Revista digital: Actividad Física y Deporte, 5(2), 171-181.

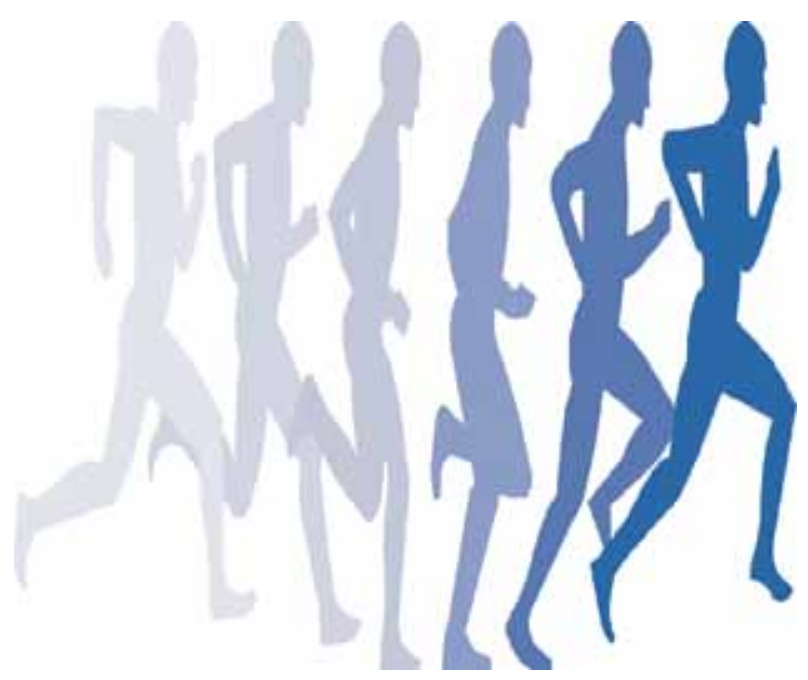

